
\section{Egatso}

${ }^{1}$ Department of Respiratory Medicine and Allergy, Kings College London, Guy's

Hospital, London, UK ${ }^{2}$ Department of Respiratory Medicine and Allergy, Kings College London, London, UK ${ }^{3}$ Department of Respiratory Medicine, Guy's \& St. Thomas' NHS Foundation Trust, London, UK

${ }^{4}$ Department of Cellular Pathology, Guy's \& St. Thomas' NHS Foundation Trust, London, UK ${ }^{5}$ Department of Radiology, Guy's \& St. Thomas' NHS Foundation Trust, London, UK ${ }^{6}$ PET Imaging Centre at St. Thomas' Hospital, King's College London, London UK

\section{Correspondence to}

Dr William T Owen, Department of Respiratory Medicine and Allergy, Kings College London, 5th Floor, Tower Wing, Guy's Hospital, London SE1 9RT, UK; williamowen@nhs.net

Received 15 February 2016 Revised 29 March 2016 Accepted 30 March 2016 Published Online First 26 April 2016

\title{
A rare intravascular tumour diagnosed by endobronchial ultrasound
}

\author{
William T Owen, ${ }^{1}$ Elena Karampini, ${ }^{2}$ Ronan A Breen, ${ }^{3}$ Mufaddal Moonim, \\ Arjun Nair, ${ }^{5}$ Sally F Barrington, ${ }^{6}$ George Santis ${ }^{1}$
}

A 24-year-old man was referred to the haematologists for investigation of unexplained anaemia on the background of a 6-month history of exertional breathlessness, mild cough and night sweats. Investigations revealed iron-deficiency anaemia (haemoglobin $94 \mathrm{~g} / \mathrm{L})$, thrombocytosis and markedly elevated inflammatory markers (C-reactive protein (CRP) $235 \mathrm{mg} / \mathrm{L}$ ). A CT scan of his chest identified a large expansile filling defect within the left main pulmonary artery, almost entirely occluding the leftsided pulmonary circulation, which had high-grade ${ }^{18}$ F-fluorodeoxyglucose (FDG) uptake on a subsequent positron emission tomography (PET) CT (figure 1).

The lesion was assessed via endobronchial ultrasound (EBUS), which identified a hyperechoic soft tissue mass within the left main pulmonary artery (figure 2). EBUS-guided transbronchial needle aspiration (TBNA) of the lesion was performed without complication and rapid on-site cytological evaluation confirmed a cellular aspirate. Subsequent cytological analysis revealed large sheets of fibrotic stroma intimately admixed with small delicate vessels, spindle-shaped cells and a prominent lymphoplasmacytic infiltrate, consistent with a diagnosis of an inflammatory myofibroblastic tumour (figure 3). The lesion was subsequently surgically excised and histological evaluation of the tumour confirmed the diagnosis. The patient has recovered well with an uncomplicated postoperative period. At 4 weeks following the resection, the anaemia had resolved $(\mathrm{Hb} 132 \mathrm{~g} / \mathrm{L})$, the platelet count was normal and the CRP had fallen to $35 \mathrm{mg} / \mathrm{L}$.
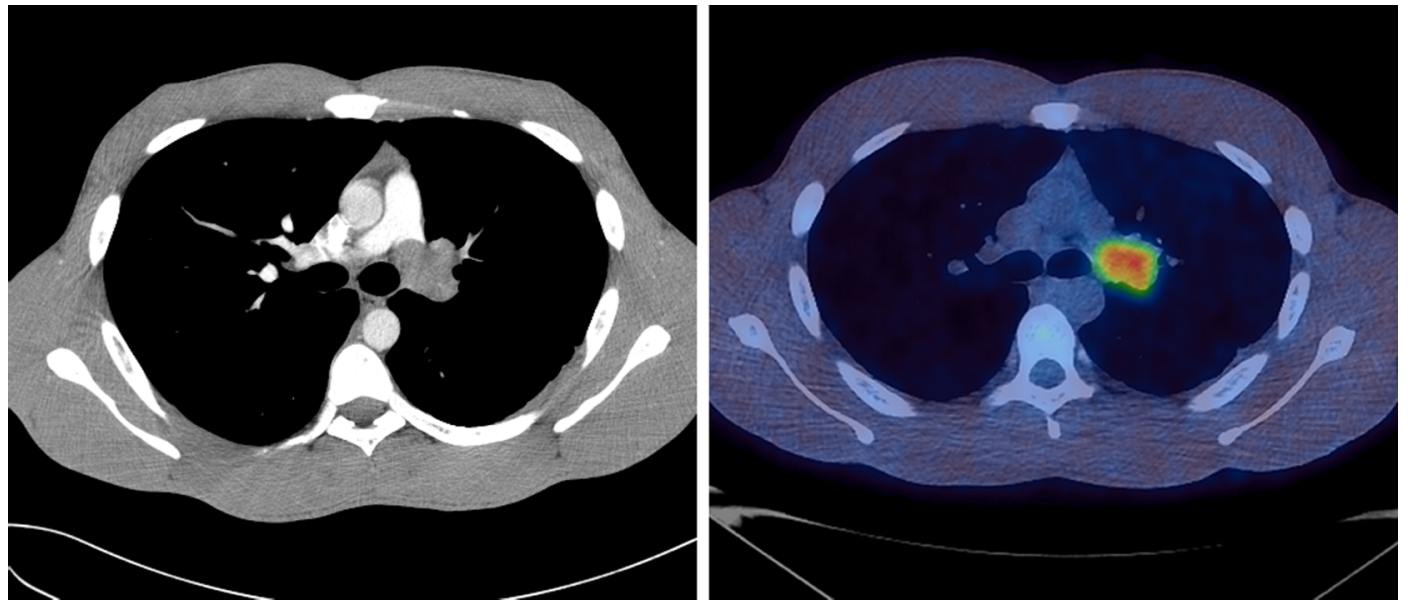

Figure 2 Endobronchial ultrasound image showing a hyperechoic soft tissue mass (M) within the left main pulmonary artery (PA).

Inflammatory myofibroblastic tumours are extremely rare and represent a spectrum of inflammatory pseudo-tumours. They occur most commonly in children and young adults, and there remains an ongoing controversy as to whether these lesions are inflammatory or truly neoplastic. They typically arise in the lung but can occur in diverse extrapulmonary locations. Surgical excision is the treatment of choice and is often the route to a final diagnosis in view of common diagnostic uncertainty following biopsy. ${ }^{1}$ In $50 \%$ of cases, chromosomal translocations of anaplastic lymphoma kinase (ALK) are present and in those patients with ALK positivity on immunohistochemical staining, crizotinib (an ALK inhibitor) has been

Figure 1 CT pulmonary angiogram and positron emission tomography CT merged image showing high-grade ${ }^{18} \mathrm{~F}$-fluorodeoxyglucose (FDG) uptake in a $35 \mathrm{~mm}$ lesion within the left main pulmonary artery. 


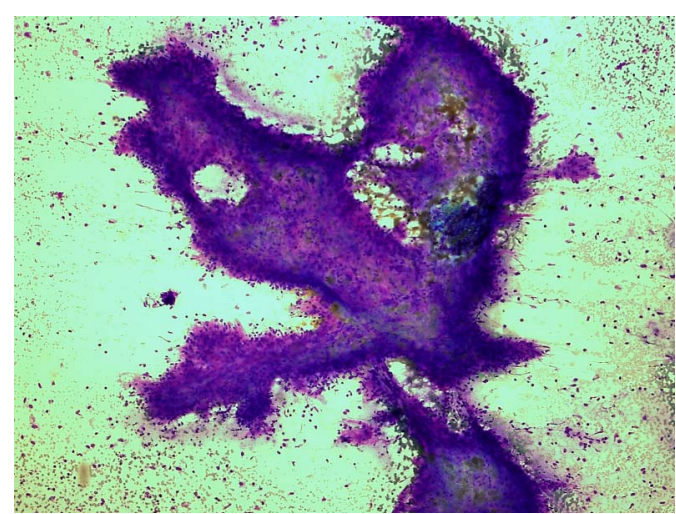

Figure 3 Scanning magnification view of microbiopsy fragments of the lesion obtained at endobronchial ultrasound identifying cytologically bland spindle cells and a variably myxoid stroma.

reported to be an effective treatment. ${ }^{2}$ Our patient's excision sample underwent fluorescence in situ hybridisation (FISH) and PCR, which identified no evidence of ALK rearrangements. Recurrence rates following complete excision of lung inflammatory myofibroblastic tumours are low, with a 5 -year survival of $>90 \% .^{3}$

Mass lesions within the pulmonary artery are rare and robust evidence supporting techniques for their assessment is therefore lacking. PET-CT scanning has been shown in a small study to provide excellent diagnostic accuracy when differentiating malignant pulmonary artery lesions from thromboembolic disease, with abnormal FDG uptake on PET being entirely indicative of a malignant lesion. ${ }^{4}$ Similarly, there is very little assessment of safety or efficacy relating to the use of EBUS-TBNA for the sampling of pulmonary artery lesions. A recent review ${ }^{5}$ identified 10 case reports of EBUS-TBNA for intravascular lesions, all of which yielded a diagnostic sample and reported no complications. This limited evidence supports the use of EBUS-TBNA for the safe diagnostic sampling of intravascular pulmonary artery lesions.

Contributors All authors wrote, revised and approved the final manuscript.

Competing interests None declared.

Patient consent Obtained.

Provenance and peer review Not commissioned; externally peer reviewed.

\section{REFERENCES}

1 Mellioni G, Carretta A, Ciriaco P, et al. Inflammatory pseudotumor of the lung in adults. Ann Thorac Surg 2005;79:426-32.

2 Butrynski JE, D'Adamo DR, Hornick JL, et al. Crizotinib in ALK-rearranged inflammatory myofibroblastic tumor. N Engl J Med 2010;363:1727-33.

3 Cerfolio RJ, Allen MS, Nascimento AG, et al. Inflammatory pseudotumors of the lung. Ann Thorac Surg 1999;67:933-6.

4 Lee EJ, Moon SH, Coi JY, et al. Usefulness of fluorodeoxyglucose positron emission tomography in malignancy of pulmonary artery mimicking pulmonary embolism. ANZ J Surg 2013;83:342-7.

5 Harris K, Modi K, Kumar A, et al. Endobronchial ultrasound-guided transbronchial needle aspiration of pulmonary artery tumors: A systematic review (with video). Endosc Ultrasound 2015;4:191-7. 